\title{
Eficacia de resolución colaborativa de problemas en el desarrollo de habilidades cognitivo lingüísticas y en el rendimiento académico en física
}

\author{
Iván R. Sánchez¹, Edith Del C. Herrera² y Carlos E. Rodríguez ${ }^{1}$ \\ (1) Universidad del Bío-Bío, Facultad de Ciencias, Departamento de Física, Avda. Collao 1202, Casilla 5-C, \\ Concepción, Chile. (correo-e: isanchez@ubiobio.cl; carlosrbfisica@gmail.com) \\ (2) Universidad del Bío-Bío, Facultad de Educación y Humanidades, Departamento de Ciencias de Educación, \\ Chillán, Chile. (correo-e: eherrera@ubiobio.cl)
}

Recibido Abr. 7, 2020; Aceptado Jun. 4, 2020; Versión final Jul. 6, 2020, Publicado Dic. 2020

\begin{abstract}
Resumen
El presente estudio tiene por finalidad reportar cómo se desarrollan las habilidades cognitivo lingüísticas (describir, explicar, argumentar y modelar matemáticamente) utilizando la resolución colaborativa de problemas para enseñar y aprender física en estudiantes de Ingeniería Civil. Se incluye el diseño, la implementación y la evaluación de la propuesta didáctica. Es de carácter cuantitativo bajo un diseño cuasi experimental de tres grupos (dos de control y uno experimental). Los resultados indican que al resolver problemas los estudiantes del grupo experimental lograron utilizar las habilidades de describir, explicar y modelar matemáticamente en mayor frecuencia que la argumentación. Se observa esto cuando los estudiantes del grupo experimental organizaron los conceptos físicos y la información en la interacción colaborativa con apoyo del profesor, al igual que su transferencia en los trabajos prácticos de laboratorio. El rendimiento académico muestra cambios estadísticamente significativos respecto a los dos grupos control. Se concluye que se puede desarrollar la competencia de resolución de problemas y algunas habilidades cognitivo lingüística.
\end{abstract}

Palabras clave: resolución colaborativa de problemas; habilidades cognitivo lingüísticas; modelamiento matemático

\section{Collaborative problem solving efficacy in cognitive linguistic skill development and in the academic performance in physics}

\begin{abstract}
This study aims to report how linguistic cognitive skills (to describe, to explain, to argue, and to model mathematically) develop in civil engineering students by using collaborative problem solving when teaching and learning physics. The present study includes the design, implementation, and evaluation of the didactic proposal. The method applied is quantitative and under a quasi-experimental design of three groups (two control groups and one experimental). The results indicate that when solving problems the experimental group students were able to use the skills of describing, explaining, and modeling mathematically more frequently than arguing. This is observed when students in the experimental group organized the physical concepts and information by collaborative interaction with the support from the professor. The experimental group students managed to transfer their skills to practical laboratory work. The academic performance in the experimental group shows statistically significant differences when compared to the two control groups. It is concluded that it is possible to develop problem solving competences and linguistic cognitive skills.
\end{abstract}




\section{INTRODUCCIÓN}

Una de las principales razones de los problemas que muchos estudiantes tienen con el aprendizaje de la física es el alto nivel de abstracción y el papel predominante de las matemáticas en la enseñanza de la física (Duit et al., 2014). La investigación ha mostrado reiteradamente que muchos estudiantes que siguen cursos de física general en la universidad en formatos de enseñanza transmisiva, aprenden a resolver problemas cuantitativos similares a los que aparecen en los libros de texto al final de los capítulos, pero suelen ser incapaces de explicar el significado de sus propias soluciones numéricas a los problemas, la instrucción habitual en estos cursos ignora importantes objetivos procedimentales y suele centrarse en un operativismo matemático (Guisasola et al., 2006; Perales,2006), por lo tanto, hay un escaso logro en el desarrollo de habilidades y procedimientos asociados a la resolución de problemas. (Guisasola et al., 2006)

Esta forma de enseñar y aprender ciencias contrasta con las demandas de la sociedad actual, las cuales apuntan a la necesidad que el área de ciencias pueda proporcionar el desarrollo de habilidades cognitivas en los estudiantes. Desde los estudios de la didáctica de las ciencias, se destaca la necesidad de apropiar los procesos investigativos en la enseñanza de la física y hacerla más contextualizada (Solbes et al., 2007), de esta manera, en lugar de seguir memorizando contenidos, los estudiantes deben desarrollar y ejercitar habilidades que les posibilitan comprender y construir sus conocimientos. Además, la mayoría de los sectores productivos exigen a los ingenieros tanto conocimientos como competencias (Schmal, 2015, y Soares et al., 2013) y habilidades especiales para su ejercicio profesional, como es el pensamiento lógico, resolución de problemas y capacidad de abstracción. Desde esta perspectiva la capacidad para resolver problemas es un componente importante de su ejercicio profesional.

El desafío de aprender a resolver problemas con el objetivo de desarrollar habilidades cognitivas no es nuevo, se vincula estrechamente con la comprensión de los enunciados y debido a la complejidad que encierra, existen diversas propuestas orientadas al qué y cómo enseñar esas habilidades. Concretamente en lo que respecta a las habilidades cognitivo lingüísticas se han realizado estudios dirigidos a identificar cuál de ellas exige el profesorado universitario en las actividades de enseñanza utilizadas en la carrera de Psicopedagogía (Vázquez et al., 2008), en formación de maestros de Primaria (Couto et al., 2013), en la meta comprensión para resolver problemas en estudiantes de Ingeniería (Sandoval y Franchi , 2007) por la falta de comprensión de los enunciados, que impide en algunos casos identificar datos, establecer metas o estrategias de solución.

El objetivo de este estudio es indagar cómo la implementación de la estrategia de resolución de problemas colaborativo a través de un método único a través del uso de la integral y derivada en cinemática y basada en el desarrollo explícito de las habilidades cognitivas lingüística describir, explicar, argumentar y modelar matemáticamente puede ayudar a los estudiantes de Ingeniería Civil de una Universidad Estatal a que un mayor número de estudiante logre el éxito (mejorar rendimiento) académico en el grupo experimental frente a grupos que siguen una enseñanza tradicional en Física General. Este enfoque resulta particularmente adecuado para el caso de los aprendizajes relacionados con la formación del ingeniero, que exige manejar la información, el planteamiento de conjeturas y una serie de acciones cognitivas que debe poner en práctica para construir sus propios conocimientos en esta área y utilizarlos para desarrollar los Trabajos Prácticos de Laboratorio de Física (TPL).

Cada sesión de clase con la propuesta didáctica se inicia analizando una situación problema por los estudiantes colaborativamente, los cuales han de describir los conceptos involucrados en el problema, explicar las relaciones entre los datos que involucra y argumentar basados en datos teóricos, empíricos para finalmente al modelar usando la integral y derivada para tomar decisiones sobre posibles vías de solución. Los estudiantes van sistematizando en la interacción grupal sus explicaciones, datos, modelos, registrando sus soluciones. Durante el desarrollo de este proceso colaborativo se realiza el seguimiento de las habilidades cognitivo lingüísticas (PISA, 2015). Los resultados indican que el rendimiento académico del grupo experimental muestra cambios estadísticamente significativos respecto a los dos grupos control, sin embargo, el estudio es relevante porque permitió develar las dificultades en la habilidad de argumentar por sobre habilidades concretas como describir, explicar y modelar matemáticamente por medio de la integral y derivada cuando los estudiantes deben realizar la organización de los conceptos físicos y datos al resolver problemas en la interacción colaborativa grupal con apoyo del profesor. Por lo tanto, concordamos con Aragón, (2007) en la idea que las habilidades cognitivo-lingüísticas están en la base de operaciones que se producen constantemente en la actividad de aprendizaje y permiten estructurar el conocimiento adquirido y el razonamiento. Su desarrollo propicia un aprendizaje más duradero, significativo y aplicable en la toma de decisiones y en la solución de problemas cotidianos.

La Organización para la Cooperación y Desarrollo Económico la define como una habilidad que considera el uso de procesos cognitivos para resolver situaciones reales en el ámbito disciplinar donde la solución no es obvia y donde los dominios de la instrucción del plan de estudios que pudieran ser aplicables no estén dentro de un solo dominio de las matemáticas, de la ciencia o de la lectura. Según su importancia, la resolución de 
problemas es una actividad central en los cursos de física en la universidad para enseñar y evaluar el aprendizaje (Nersessian, 1995). En este contexto, la resolución de problemas debe considerar que quien resuelve el problema debe comprender, caracterizar, representar, reflexionar y comunicar lo obtenido.

\section{OTROS ANCEDENTES}

Hay otros antecedentes que es necesario presentar y discutir en el contexto de este trabajo: i) la resolución de problemas de Física y estrategias de aprendizaje; y (ii) las habilidades cognitivo lingüísticas. Estas se analizan en lo que sigue.

\section{La resolución de problemas de Física}

En la enseñanza de física la resolución de problemas se utiliza como estrategia para demostrar el logro de los estudiantes (Docktor et al., 2016; Ceberio et al., 2016; Adams y Wieman, 2015). Además, una buena habilidad para resolver problemas podría ayudar a los estudiantes a transferir su conocimiento y comprender la situación física (Walsh et al., 2007). Cuando alguien intenta resolver un problema, hará un modelo en su mente sobre cómo resolver ese problema (Khasanah et al., 2016), porque gracias a este modelo podría mejorar la capacidad de los estudiantes para resolver problemas (Supriyanti et al., 2015). Para Truyol et al., (2014) es relevante conocer los obstáculos cognitivos que los estudiantes encuentran al resolver diferentes problemas en física, ya que su modelamiento involucra procesos cognitivos de alta complejidad, de esta manera se podrían diseñar materiales y métodos de instrucción más efectivos que fomenten la capacidad de los estudiantes para conectar la realidad con los principios, leyes y conceptos de Física, así como también usar la lógica y las matemáticas para formalizar la Física, de modo de alcanzar mejor los objetivos de la educación científica

De acuerdo con Docktor et al., (2015), hay dos enfoques utilizados por los estudiantes en la resolución de problemas; resolución explícita de problemas y solución tradicional de problemas. Los estudiantes que usan la resolución explícita de problemas generalmente resuelven el problema enfocándose en él, describiendo el concepto de física que se aplicará, planificando la solución, terminando el plan y evaluando la solución, mientras que los estudiantes que usan la solución tradicional de problemas comenzarán a dibujar bocetos, definiendo una variable conocida y desconocida, eligiendo la ecuación y corrigiendo la respuesta. Según Sánchez et al. (2015) la resolución de problemas se realiza a través del uso de la integral y derivada como modelo único que favorece la interpretación física y el reconocimiento de condiciones de frontera iniciales y finales. Esta forma de abordar los contenidos de cinemática promueve la habilidad de interpretar, describir y transferir los conocimientos adquiridos en nuevas situaciones, lo que se logra por medio de la comprensión de lo que realiza.

Resolver problemas de forma colaborativa brinda importantes oportunidades de aprendizaje y desarrollo de competencias, sobre todo cuando los problemas demandan altos niveles de interdependencia positiva (Johnson, et al., 1986), esto motiva la participación de quienes interactúan para ayudarse a buscar explicaciones, al resolver la tarea, deben compartir la creencia de que el éxito es fruto del trabajo conjunto y la responsabilidad es tanto individual como grupal y desde donde se hace relevante destacar la importancia de la colaboración desde la docencia (Pulgar, Candia y Leonardi, 2020).

Por su parte la OCDE, en PISA (2015) dio cuenta de una nueva área de evaluación de competencias, la resolución colaborativa de problemas (CPS, por sus siglas en inglés, collaborative problem solving) definiéndola como la capacidad de un individuo para involucrarse de manera efectiva en un proceso a través del cual dos o más agentes intentan resolver un problema compartiendo la comprensión y el esfuerzo necesario para llegar a una solución y aunando sus conocimientos, destrezas y esfuerzos para este fin. Los componentes cognitivos de la resolución de problemas abordan la comprensión y representación del contenido del problema, la aplicación de estrategias para resolverlos. La definición de resolución colaborativa de problemas (CPS) incorpora tres competencias claves, con las destrezas asociadas a cada una de ellas que muestra la Figura 1.

En la resolución colaborativa de problemas se ponen en marcha la combinación de estos procesos de forma individual y colaborativa, cuyo fortalecimiento se hace necesario en la formación de los estudiantes en la educación en ingeniería. Cuando los estudiantes leen un problema, la percepción y la significatividad que este adquiere se expresa de forma oral o por escrito, la comprensión de un texto a través de la lectura requiere de habilidades cognitivas lingüística y estas a su vez requieren previamente haber desarrollado habilidades cognitivas básicas como: analizar, comparar, clasificar, identificar, interpretar, inferir, deducir, transferir, etc. Por esta razón, es que las teorías socioculturales constructivistas del aprendizaje han destacado el papel del lenguaje, en la adquisición de habilidades cognitivas lingüísticas ya que el diálogo permite la negociación de significados (Perales y Cañal, 2000). 
Resolución colaborativa de problema (CPS)

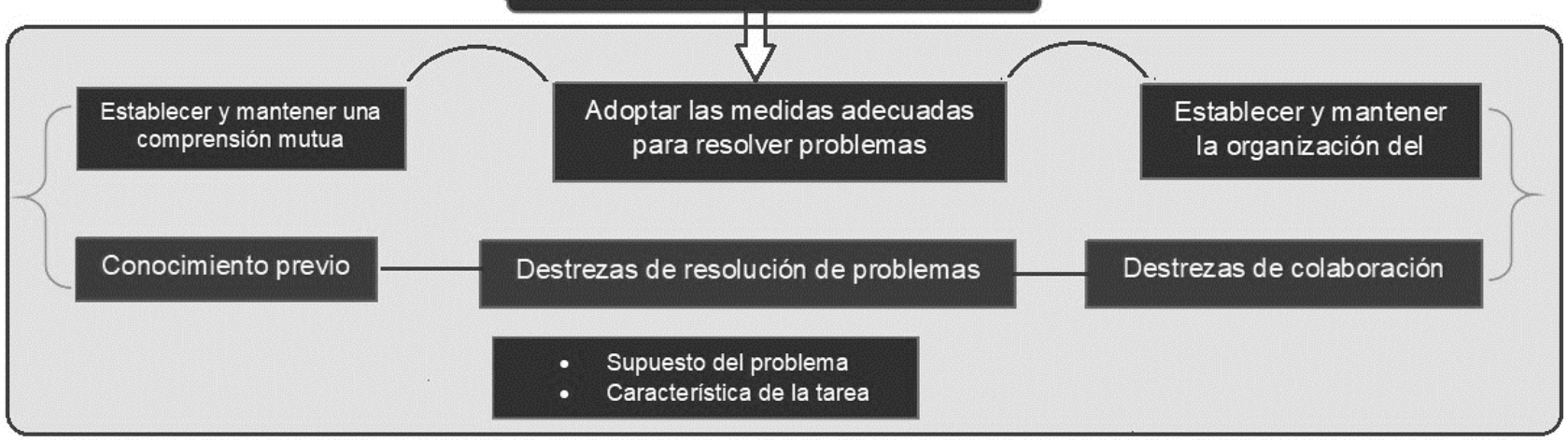

Fig. 1: Competencias claves y destrezas según PISA ,2015 en la resolución colaborativa de problemas.

\section{Las habilidades cognitivo lingüísticas (HCL)}

Para acercarnos al concepto habilidades cognitivo lingüísticas (HCL), se requiere considerar dos elementos claves: las habilidades cognitivas y las habilidades lingüísticas y el modo en cómo estas se relacionan. De acuerdo con Jorba et al., (2000) Las habilidades cognitivo-lingüísticas, como describir, definir, resumir, explicar, justificar y argumentar se derivan del uso de las habilidades cognitivas analizar, comparar, clasificar, identificar, interpretar, inferir, deducir, transferir, etc. Son estas habilidades las que reclaman y activan las $\mathrm{HCL}$ cuando se usan para comunicar información y lo que se pueden evidenciar en los trabajos de los estudiantes, en la producción de textos de diferente naturaleza, características y estructura, e influyen en la construcción de conceptos, modelos y teorías. Por tanto, se considera imprescindible la enseñanza de estas habilidades cognitivo lingüísticas para la correcta expresión y comunicación de las ideas que toman cuerpo cuando se ha apropiado del lenguaje científico.

Las habilidades cognitivo lingüístico involucran un conjunto de conceptos y procedimientos lingüísticos que permiten el desarrollo de competencias discursivas y al mismo tiempo, la construcción del conocimiento. Los estudios sobre el perfil de un profesional del siglo XXI señalan como esencial el desarrollo de las habilidades y competencias cognitivas (Pirela y Cortés, 2014) para desempeñarse en trabajos que hoy no existen, aprendiendo a renovar continuamente una parte importante de sus conocimientos, adquiriendo nuevas competencias en el manejo de información, de comunicación, resolución de problemas, trabajo en equipo, entre otras (21st Centurry Skills, 2002). Estas son capacidades innatas de la mente humana utilizadas para el razonamiento; el hecho de ser innatas, indica que están presentes y pueden cambiar o no con el tiempo (Mayer et al., 2008). Estas habilidades son rutinas cognitivas existentes y empleadas para facilitar la adquisición y producción del conocimiento; son las destrezas y procesos necesarios para realizar una tarea, además son las facilitadoras del conocimiento al ser las responsables de adquirirlo y recuperarlo para utilizarlo posteriormente (Reed, 2007).

Para adquirir una habilidad cognitiva según Ramos et al. (2010) es preciso que el estudiante ejecute tres momentos específicos que son: i) el reconocimiento, en la que aún no se es capaz de aplicar el conocimiento, ii) el desarrollo, en la que puede aplicar un sólo principio o varios de ellos y iii) la de ejecución en la que realizan las acciones sin errores y puede ser utilizada como estrategia posteriormente de forma independiente. En el profesor recae la tarea de enseñar las habilidades cognitivas en general y las cognitivo lingüísticas en particular a través de actividades específicas en el aula y a pesar de su importancia, desde la enseñanza de las ciencias estas habilidades no se han desarrollado suficientemente.

La adquisición de habilidades cognitivo lingüísticas no se realiza de manera independiente, sino que se relaciona con el desarrollo de habilidades cognitivas y el aprendizaje de contenidos curriculares, así el estudiante tendrá que describir los hechos/fenómenos y los modelos teóricos que exige la ciencia, pues una buena descripción es la base necesaria para poder elaborar otros tipos de textos, como definiciones o justificaciones (García y Martínez, 2014).En este sentido existe consenso en estos autores sobre "enseñar a pensar", para que el estudiante desarrolle capacidades y habilidades cognitivas que ayudan a reducir el fracaso y las bajas calificaciones; modificando esta forma de aprender se evita una simple recepción de conocimientos o la resolución mecánica de problemas como señalan Pérez-Sánchez y Poveda-Serra (2008).

Desde la perspectiva de Córdoba et al. (2018) promover el desarrollo de habilidades cognitivas como, por ejemplo: identificar, analizar, interpretar, sintetizar, deducir, transferir e inferir, facilitará posteriormente el desarrollo de habilidades cognitivas lingüísticas para comunicarse, con las cuales los estudiantes podrán 
describir, explicar justificar y argumentar, diferentes fenómenos naturales, de esta forma estas habilidades se potencian entre sí. Jorba et al. (2000) ya habían realizado una asociación entre la comunicación, producida mediante diferentes tipologías textuales, y las habilidades cognitivas que se activan, refiriéndose así a las habilidades cognitivo lingüísticas. Basándonos en esta progresión y diferenciación según niveles crecientes de complejidad de las habilidades cognitivo lingüísticas se presenta en la tabla1. Se ha incorporado a la clasificación original, el modelar matemáticamente por los autores a esta tabla.

Tabla 1: Nivel de complejidad de habilidades cognitivo lingüísticas según Jorba et al. $(2000: 34,37,39$ y 40)

\begin{tabular}{|l|l|l|}
\hline Niveles & Habilidad & Descripción \\
\hline Concreto - simple & Describir & $\begin{array}{l}\text { Consiste en producir proposiciones o enunciados que enumeran cualidades, propiedades, etc., } \\
\text { de objetos, hechos o fenómenos o incluso de modelos explicativos abstractos (modelo } \\
\text { corpuscular de la materia, modelo de célula }\end{array}$ \\
\cline { 2 - 3 } & Explicar & $\begin{array}{l}\text { Presentar razonamientos o argumentos estableciendo relaciones (debe haber relaciones } \\
\text { causales explícitamente) en el marco de las cuales los hechos, acontecimientos o cuestiones } \\
\text { explicadas adquieren sentido y llevan a comprender o a modificar un estado de conocimiento. }\end{array}$ \\
\hline Abstracto - complejo & Justificar & $\begin{array}{l}\text { Implica elaborar la expresión de una interpretación de hechos, fenómenos o acontecimientos } \\
\text { vinculándolos con la teoría. }\end{array}$ \\
\cline { 2 - 3 } & Argumentar & $\begin{array}{l}\text { Requiere construir los enunciados en base a pruebas con la intención de ser empleadas para } \\
\text { convencer a otras personas. Para ello, es necesario integrar la habilidad de explicar con la de } \\
\text { justificar y trabajar con contra-ejemplos para sostener o refutar las objeciones en un } \\
\text { determinado punto de vista con las ideas de otro. }\end{array}$ \\
\cline { 2 - 3 } & $\begin{array}{l}\text { *Modelar } \\
\text { matemáticamente }\end{array}$ & $\begin{array}{l}\text { Modelo de representación matemática que describe, explica y predice aproximadamente los } \\
\text { efectos del fenómeno real que se desea representar (Lamon et al., 2003). Incluye el proceso de } \\
\text { metareflexión, un análisis crítico de las relaciones establecidas entre el modelo - realidad y } \\
\text { viceversa. }\end{array}$ \\
\hline
\end{tabular}

Las teorías socioculturales constructivistas del aprendizaje han destacado el papel del lenguaje, en la adquisición de habilidades cognitivas ya que el diálogo permite la negociación de significados (Perales y Cañal, 2000). Cuando los estudiantes leen un problema la percepción y la significatividad que este adquiere se expresa de forma oral o por escrito, la comprensión de un texto a través de la lectura requiere de habilidades cognitivo lingüística y estas a su vez necesitan que se hayan desarrollado habilidades cognitivas básicas. En este sentido según Jorba et al. (2000) el alumno deberá emplear textos diferentes conjugando habilidades cognitivas, con habilidades cognitivo lingüísticas. La investigación ha abordado cómo los estudiantes articulan y justifican sus conclusiones o explicaciones entre otros, Lawson, 2002; Zohar y Nemet, 2002, así como el proceso por medio del cual los estudiantes interaccionan entre ellos cuando proponen, evalúan y critican ideas entre otros Kuhn y Reiser, 2006; Osborne et al., 2004. El análisis de los argumentos elaborados por los estudiantes puede aportar información importante acerca de la comprensión de los contenidos científicos alcanzada (por ejemplo, las teorías, leyes y principios de la física), el razonamiento científico de los estudiantes, los aspectos epistemológicos (por ejemplo, qué cuenta como justificación en ciencias) y la habilidad de los estudiantes para comunicar y justificar ideas.

Considerando los referentes revisados en este estudio entendemos las habilidades cognitivo lingüísticas como las operaciones mentales que el estudiante utiliza para aprender en una situación dada y nos centraremos en las habilidades de describir, explicar, argumentar y modelar matemáticamente, para diferenciarlas en ciencias de las habilidades de pensamiento científico que incluyen: observar, plantear hipótesis, identificar y combinar variables, diseñar experimentos, recoger datos y transformarlos, y concluir.

\section{METODOLOGÍA}

La investigación responde a un enfoque cuantitativo a través de un diseño cuasi-experimental de tres grupos independientes (Cohen y Manion, 1990), un Grupo experimental (GE) y dos grupos control (GC) con pre y post-test en la variable de estudio: rendimiento académico en la carrera de Ingeniería Civil de la Universidad del Bío-Bío. Todos los grupos cursan la asignatura Física I, bajo estructura modular.

La estructura modular de la asignatura de física implicó un rediseño curricular que permitió organizar productos y procesos complejos de una manera eficiente y organizó dos módulos en los que se incluyen unidades de aprendizaje más reducidas a aprender, antes de acceder al siguiente módulo. Los alumnos pueden reprobar un solo modulo del curso, y tienen la posibilidad de cursar el módulo II al final del semestre de forma intensiva. La asignatura es certificada cuando ambos módulos se aprueban de forma independiente. El módulo I agrupa los contenidos de cinemática y dinámica y el módulo II trabajo, energía, principios de conservación y dinámica de cuerpo rígido. De esta forma se espera desarrollar en los estudiantes la competencia específica de: i) Aplicar las leyes que explican el movimiento de los cuerpos, utilizando los modelos de partículas y cuerpo rígido en la solución de problemas y ii) Resolver problemas de movimiento de partículas al finalizar ambos módulos del curso. 
El estudio se organizó en dos fases, la fase I se desarrolló partir de la revisión teórica que permitió la elaboración del diseño de las situaciones problema de la propuesta didáctica por cuatro docentes de Física, las cuales fueron organizadas y estructuradas para el aprendizaje colaborativo de los conceptos de física ya mencionados del módulo I y II con el objetivo de promover en los estudiantes el desarrollo de las habilidades de describir, explicar, argumentar y modelizar matemáticamente. Posteriormente, se solicitó la validación de los problemas y la rúbrica de evaluación utilizada por juicio de tres expertos.

Se realizó un estudio piloto exploratorio con 22 estudiantes voluntarios que cursaron la asignatura en el primer semestre 2018 a quienes se les informó del propósito de analizar cualitativamente la comprensión de las situaciones problemáticas. Se procuró que los enunciados no presentasen dificultades léxicas, ni ambigüedades sintácticas, redactados de la manera más clara y comprensible posible. La totalidad de los estudiantes estuvieron de acuerdo que la redacción de los problemas era clara, identificaron a que conceptos correspondían con sus significados y expresaron sus dificultades al realizar el modelamiento matemático. Como resultado de esta fase se obtuvo la adecuación de las situaciones problema y de los instrumentos evaluativos, realizando los ajustes necesarios para luego ser empleados en la recolección de información La fase II. Corresponde a la implementación en clases de la asignatura modular de física de la propuesta de cambio didáctico en la teoría y trabajo práctico laboratorio (TPL) durante el año 2018. Los estudiantes en las primeras clases recibieron una inducción en la estrategia de cambio didáctico. Trabajaron en grupos colaborativos analizando la situación problema con la guía del profesor y se realizaron en cada módulo dos evaluaciones de ciclo. Estas pruebas de ciclo se denominan así, porque han sido construidos en conjunto por los docentes y validadas por los distintos profesores que imparten la asignatura. El rendimiento académico, como variable de estudio se obtiene del promedio alcanzado en las evaluaciones de ambos módulos.

La muestra que participó en este estudio estuvo conformada por 160 estudiantes de primer año de la carrera de Ingeniería Civil, de la Universidad del Bío-Bío (población) del semestre Il elegidos en muestreo intencional, por conveniencia y clasificados por grupos de estudio como muestra la Tabla 2. Antes de comenzar la intervención didáctica, el profesor entregó y retiró el consentimiento informado de los estudiantes del GE y se explicaron los objetivos de la innovación metodológica de aula.

Para la recolección de datos de esta investigación se utilizaron como instrumentos y técnicas de medición: 1) Situación problema que explicitan las habilidades cognitivo lingüísticas, 2) evaluaciones de pruebas escritas, que entregaron información del proceso de enseñar y aprender las habilidades cognitivo lingüísticas. 3) evaluación de informes de TPL de Física 4) Rúbrica de evaluación. Las habilidades cognitivo lingüísticas se evaluaron tanto en la teoría como en la práctica en los indicadores que presenta la Tabla 3, siguiendo la recomendación dada por Jorba et al. (2000) y Córdoba et al. (2018). A través, de esta rúbrica se estableció el uso correcto cuando el estudiante alcanzó nivel de logro (3) de la habilidad en la resolución de situaciones problemas en las clases de teoría y TPL de Física, y se procedió a la tabulación de los datos en una hoja de cálculo computarizada, bajo el programa SPSS versión 20, con el cual se realizó el análisis de la información, mediante estadística descriptiva no paramétricas con la prueba de $U$ de Mann-Whitney y Wilcoxon para comparar los promedios de los dos GC y el GE en dos mediciones.

Tabla 2: Datos de los participantes del estudio de resolución colaborativa de situaciones problemáticas

\begin{tabular}{|l|l|l|}
\hline Grupos Ingeniería Civil & Asignación & Tratamiento \\
\hline Grupo Experimental $(\mathrm{n}=40)$ & No azar & $\begin{array}{l}\text { Secuencia didáctica en resolución de problemas y } \\
\text { habilidades cognitivo lingüísticas: describir, explicar, } \\
\text { argumentar y modelizar matemáticamente. }\end{array}$ \\
\hline $\begin{array}{l}\text { Grupo Control } 1(\mathrm{n}=60) \\
\text { Grupo Control } 2(\mathrm{n}=60)\end{array}$ & No azar & Clases de Física Tradicional \\
\hline
\end{tabular}

\section{PROPUESTA DIDÁCTICA}

La propuesta didáctica que se ha diseñado, la que se fundamenta en la resolución de situaciones problema de Física. La selección de los problemas se ha realizado de modo que se cumplan los objetivos especificados en el programa de Física I, incluyendo problemas de cinemática, dinámica y energía, tales como: problemas de lanzamiento de proyectil, plano inclinado de fuerza, choques elásticos e inelásticos, caída libre, correspondiente al estudio de la cinemática, dinámica y energía para una intervención de seis horas de clase teóricas a la semana y dos de laboratorio. Para ilustrar la forma de abordar la resolución de problemas para el desarrollo de las habilidades cognitivas lingüísticas desde la física, se ilustran con un ejemplo resuelto en el aula por uno de los grupos colaborativos de estudiantes de Ingeniería Civil. 
Tabla 3: Rúbrica de evaluación del desarrollo de habilidades cognitivo lingüísticas en estudiantes Ingeniería del GE

\begin{tabular}{|l|l|l|}
\hline Hab. & Descriptor: el estudiante es capaz de... & Nivel \\
\hline \multirow{4}{*}{ Describir } & Escribe sus ideas sobre características, datos y relaciones de la situación problema & 1 \\
\cline { 2 - 3 } & Representa conocimientos físicos suficientes en la Situación Problema (S.P) & 2 \\
\cline { 2 - 3 } & Identifica en su texto lo esencial de sus propiedades físicas de la S.P & 3 \\
\hline \multirow{4}{*}{ Explicar } & Explicita razones y establece relaciones entre ellas (causa-efecto) en la S.P & 1 \\
\cline { 2 - 3 } & Los conocimientos de física representados en el dibujo de la S.P son aceptables & 2 \\
\cline { 2 - 3 } & Da razones que evidencian cómo se comprende la situación problema & 3 \\
\hline & $\begin{array}{l}\text { Explicita razones y establece relaciones entre ellas (causa-efecto), estas son aceptables } \\
\text { dentro del corpus de conocimiento físico para esa S.P }\end{array}$ & 1 \\
\cline { 2 - 3 } & $\begin{array}{l}\text { Enunciar razones con la intención de modificar su estado de conocimiento en relación con el } \\
\text { corpus de conocimiento de física sobre la situación problema }\end{array}$ & 2 \\
\cline { 2 - 3 } & $\begin{array}{l}\text { Utiliza las explicaciones y razonamientos desde su valoración personal a partir de los } \\
\text { conocimientos que se tienen desde la física sobre la S.P }\end{array}$ & 3 \\
\hline Modelar \\
Matemática & Describe las variables físicas involucradas en el modelo del problema & 1 \\
\cline { 2 - 3 } & Hace Uso correcto del lenguaje matemático, datos , unidades & 3 \\
\cline { 2 - 3 } & Expresa adecuadamente la solución al problema y evalúa las respuestas. \\
\hline
\end{tabular}

Un jugador de béisbol logra batear la pelota con una rapidez de $20[\mathrm{~m} / \mathrm{s}]$ y un ángulo de $10^{\circ}$ con respecto a la horizontal. Un segundo jugador ubicado a $30[\mathrm{~m}]$ del origen, debe atrapar la pelota (Figura 2). Para ello el sujeto acelera constantemente y logra atrapar la pelota a $21[\mathrm{~m}]$ del origen. ¿Cuál es la aceleración y velocidad del segundo jugador cuando toma la pelota? y ¿Cuál es el tiempo de vuelo de la pelota y su velocidad final al ser atrapada?, Recomendación, ubique su sistema de referencia y analice condiciones del aire y terreno donde están los jugadores y asigne valores iniciales y condiciones para alcanzar la solución del problema.

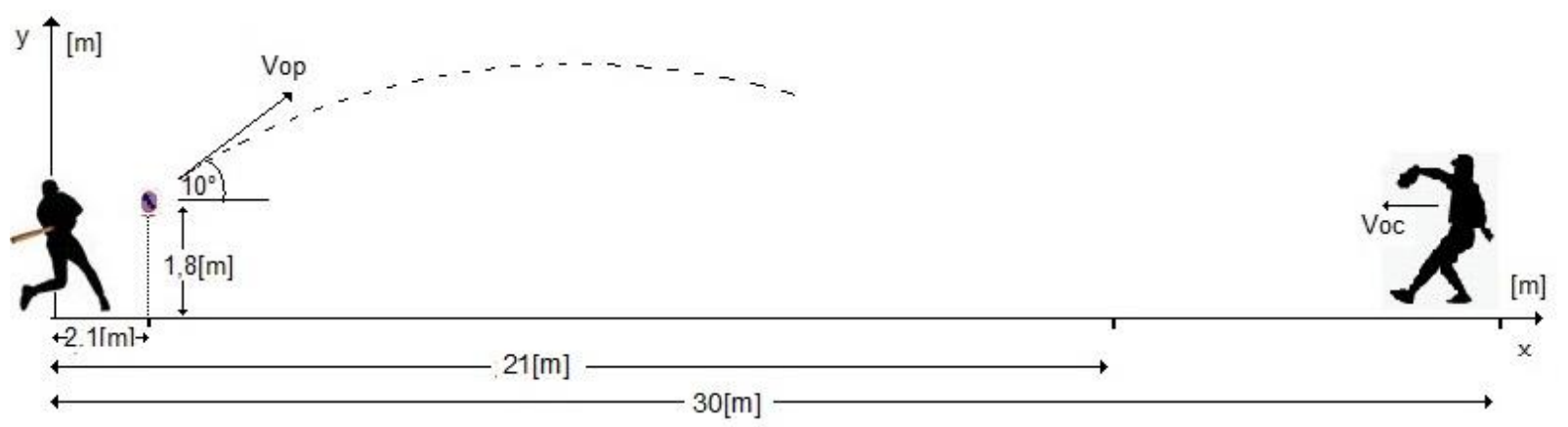

Fig. 2: Lanzamiento de una pelota de béisbol

\section{Describir}

El jugador batea la pelota con una rapidez de $20[\mathrm{~m} / \mathrm{s}]$ sobre un ángulo de $10^{\circ}$ con respecto a la horizontal; La trayectoria que describe la pelota es parabólica; La pelota al moverse por su trayectoria, acelera. / (falta indicar que la aceleración solo afecta al movimiento vertical); La pelota logra un alcance y llega a la posición de 21 [m]. / (Aquí el vector posición es $21 \hat{i}+0 \hat{j}$, el alcance es 21[m]); El jugador ubicado a 30[m] del origen, corre a atrapar la pelota, desde del reposo. / (debe considerar la aceleración)

\section{Explicar}

La trayectoria de la pelota es parabólica, esto es, cambia su dirección de movimiento y su velocidad, en dirección y módulo, en cada instante de tiempo; La pelota realiza un movimiento uniformemente acelerado. / (debiera incluirse movimiento en dos dimensiones); El jugador que atrapa la pelota, se mueve con un movimiento Rectilíneo Uniformemente Variado.

\section{Argumentar}

Una vez que el jugador lanza la pelota con una rapidez de $20[\mathrm{~m} / \mathrm{s}]$ y con un ángulo de $10^{\circ}$, esta describe una trayectoria parabólica debido a la aceleración de la gravedad y se desprecia la fricción del aire. / (que afecta al movimiento vertical); El otro jugador parte desde el reposo y corre en sentido opuesto a la pelota, así va al encuentro de la pelota, de tal manera que logra atrapar la pelota, acelerando para esto. / (empleando el mismo tiempo de vuelo de la pelota en su recorrido para atraparla) 


\section{Modelar matemáticamente}

Las ecuaciones para obtener la posición y la velocidad son: $\vec{V}(t)=\int \vec{a}(t) d t ; \vec{r}(t)=\int \vec{V}(t) d t$

\section{Solución del Problema}

Para la pelota las ecuaciones particulares encuentran usando las condiciones iniciales que siguen.

$$
\begin{aligned}
& \vec{r}(0)=(0 \hat{i}+1,8 \hat{j})[\mathrm{m}] \\
& \vec{v}(0)=(20 \cdot \cos 10 \hat{i}+20 \cdot \operatorname{sen} 10 \hat{j})\left[\frac{m}{s}\right] \approx 19,7 \hat{i}+3,5 j[\mathrm{~m}] \\
& \overrightarrow{\mathrm{a}}(0)=\overrightarrow{\mathrm{a}}(\mathrm{t})=(0 \hat{\mathrm{i}}-9,8 \hat{\mathrm{j}})\left[\mathrm{m} / \mathrm{s}^{2}\right] \\
& \vec{v}(t)=\int \vec{a}(t) \cdot d t=\int-9,8 \hat{j} \cdot d t=-9,8 \hat{j} \cdot t+c \Rightarrow \vec{v}(0)=(19,7 \hat{i}+3,5 \hat{j})\left[\frac{m}{s}\right]=c \\
& \vec{v}(t)=19,7 \hat{i}+(3,5-9,8 \cdot t) \hat{j}\left[\frac{m}{s}\right] \\
& \vec{r}(t)=\int \vec{v}(t) \cdot d t \Rightarrow \vec{r}(t)=\int[19,7 \hat{i}+(3,5-9,8 \cdot t) \hat{j}] d t \\
& \vec{r}(t)=19,7 \hat{i} \cdot t+\left(3,5 \hat{j} \cdot t-4,9 \hat{j} \cdot t^{2}+c\right) \Rightarrow \vec{r}(0)=(0 \hat{i}+1,8 \hat{j})[m]=c \\
& \vec{r}(t)=\left[19,7 \hat{i} \cdot t+\left(1,8 \hat{j}+3,5 \hat{j} \cdot t-4,9 \hat{j} \cdot t^{2}\right)\right][m] \quad \Rightarrow \\
& r_{x}(t)=19,7 \hat{i} \cdot t[m] \wedge \quad r_{y}(t)=\left(1,8+3,5 \cdot t-4,9 \cdot t^{2}\right) \hat{j}[m]
\end{aligned}
$$

Las ecuaciones particulares del movimiento del jugador se encuentran usando el modelo matemático y condiciones iniciales de este, que son:

$$
\begin{aligned}
& \vec{r}(0)=(30 \hat{i}+0 \hat{j})[m] \\
& \vec{v}(0)=(0 \hat{i}+0 \hat{j})\left[\frac{m}{s}\right] ; \\
& \vec{a}(0)=a(t)=-(a \hat{i})\left[m / s^{2}\right] \\
& v(t)=\int \vec{a}(t) \cdot d t=\int-a \hat{i} \cdot d t=-a \hat{i} \cdot t+c \Rightarrow \vec{v}(t)=a \hat{i} \cdot t[m / s] \\
& \vec{r}(t)=\int \vec{v}(t) \cdot d t=a \hat{i} \cdot t \cdot d t[m] \Rightarrow \vec{r}(t)=\left(30+\left(\frac{a i \cdot t^{2}}{2}\right)\right) \hat{i}[m]
\end{aligned}
$$

La velocidad final de la pelota se encuentra con la condición final de su posición para tiempo de vuelo.

$$
\begin{aligned}
& \vec{r}(t v)=(21 \hat{i}+0 \hat{j})[m] \\
& (21 \hat{i}+0 \hat{j})[m]=\left[19,7 \hat{i} \cdot t+\left(1,8 \hat{j}+3,5 \hat{j} \cdot t-4,9 \hat{j} \cdot t^{2}\right)\right][m] \Rightarrow 21 \hat{i}=19,7 \hat{i} \cdot t_{v} \Rightarrow \frac{21}{19,7}=t \\
& \Rightarrow 1,06[s]=t_{v} \Rightarrow \vec{v}(t)=19,7 \hat{i}+(3,5-9,8 \cdot 1,06) \hat{j}\left[\frac{m}{s}\right] \Rightarrow \vec{v}(t)=19,7 \hat{i}-6,88 \hat{j}\left[\frac{m}{s}\right]
\end{aligned}
$$


La velocidad final del segundo jugador se encuentra con las condiciones finales posición y tiempo de vuelo. Aquí el estudiante debe cuestionar la respuesta como el jugador pueda alcanzar tan alta velocidad, que condiciones físicas llevarían a lograr esta velocidad en la realidad.

$$
\begin{aligned}
& \vec{r}(t v)=(21 \hat{i}+0 \hat{j})[m]_{\wedge} t_{v}=1,06[\mathrm{~s}] \\
& 21=30-\frac{a \cdot(1,06)^{2}}{2} \Rightarrow-9=\frac{a \cdot 1,12}{2} \Rightarrow \frac{-18}{1,12}=a \Rightarrow-16.07\left[\mathrm{~m} / \mathrm{s}^{2}\right]=a \\
& \vec{v}(t)=-16,07 \hat{i} \cdot 1,06[\mathrm{~m} / \mathrm{s}]=-17,03 \hat{i}[\mathrm{~m} / \mathrm{s}]
\end{aligned}
$$

\section{RESULTADOS}

Para una mejor presentación los resultados se exponen en dos subsecciones: (i) resolución de problemas y rendimiento académico; y (ii) resolución de Problemas y desarrollo de habilidades cognitivo lingüísticas

i) Resolución de problemas y rendimiento académico.

Los resultados obtenidos del análisis del rendimiento académico del GC1, GC2 y del GE al final del semestre después de haber rendido diversas pruebas durante el semestre se muestran en la Figura 3. Se hace notar que antes de la intervención no se encontraron diferencias estadísticamente significativas entre los GC1, GC2 y GE en el test de conocimientos previos; en cambio al revisar los rendimientos después de las respectivas intervenciones al final del semestre, se encuentran diferencias en el rendimiento académico a favor del GE. Lo anterior, se corrobora por medio de la prueba $U$ de Mann Whitney que muestra diferencias estadísticamente significativas a favor del GE con un nivel de significados $p=0,000$ (99,99, \%).

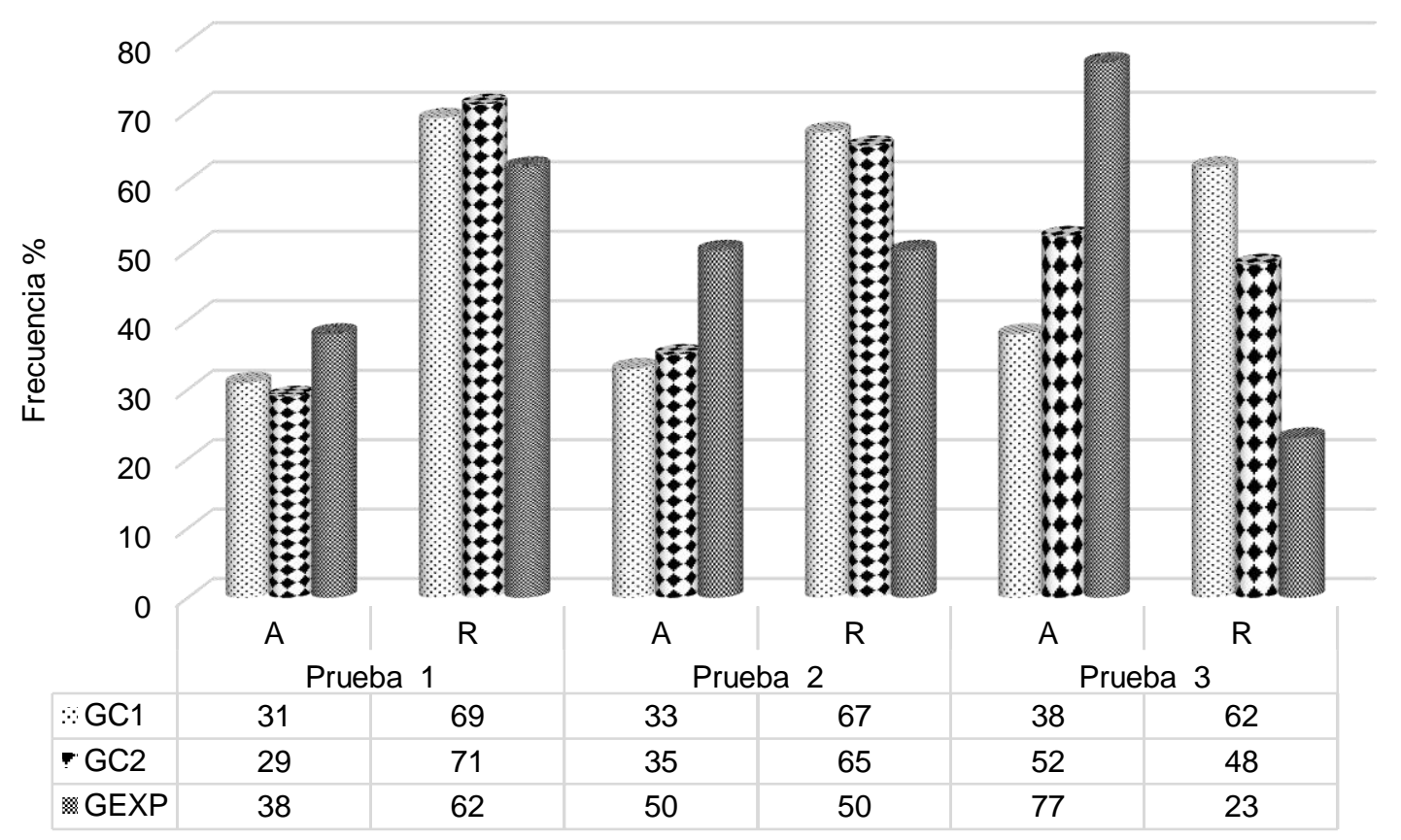

Fig. 3: Rendimiento académico de estudiantes de Ingeniería Civil por grupos.

En la Figura 3, se deduce un bajo rendimiento académico ( $A$ : aprobado y $R$ : reprobado) en la primera medición, en el GC1 y GC2 los aprobados (A) son 31\% a 29\% respectivamente y en el GE un $38 \%$. Estas frecuencias son similares en la segunda medición para el GC1 y GC2, en cambio en el GE los aprobados (A), alcanzan al 50\%. En la tercera medición los aprobados en los grupos son: GC1 un 38\%; GC2 un 52\% y GE un $77 \%$, lo que indica que la propuesta didáctica implementada impacta positivamente al rendimiento académico. Este estudio indica que los participantes del GE mejoraron sus habilidades cognitivo lingüística del nivel concreto simple (Córdoba et al, 2018), las cuales son necesarias para la formación de los futuros ingenieros, porque deberán aprender de fuentes formales e informales a resolver de manera creativa y efectiva problemas complejos. 


\section{ii) Resolución de Problemas y desarrollo de habilidades cognitivo lingüísticas}

El resultado obtenido de las actividades de resolución de problema en la teoría se muestra en la Figura 4, que evidencia una mejora en el desempeño del uso de las habilidades cognitivo lingüísticas. En la Figura 4 se infiere que los estudiantes de Ingeniería desarrollan las dos primeras habilidades de describir y explicar alcanzando un logro aproximado de un $80 \%$ en ambas, similar al modelado matemático $80 \%$, en menor medida se desarrolla la habilidad de argumentar (70\%) durante su trabajo colaborativo en las clases. Resultados similares a la teoría se obtienen en el uso de las habilidades cognitivo lingüísticas al resolver situaciones problema en los trabajos prácticos de laboratorios (TPL), que se presentan en la Figura 5 , donde se evidencia el uso del describir, explicar, argumentar y modelar.

En la Figura 5, se infiere que en los TPL se desarrollan en mayor frecuencia la habilidad de describir y explicar, en menor frecuencia la argumentación, el modelamiento matemático mejora como avanzan las actividades de aula y laboratorio resultados similares a los alcanzados en la teoría. Para establecer el desempeño alcanzado por el GE en cada habilidad cognitivo lingüística estudiada se utilizó la rúbrica antes presentada, la Figura 6 presenta los resultados del seguimiento realizado al nivel desarrollo de las habilidades cognitivo lingüísticas: describir, explicar, argumentar y modelar matemáticamente en las situaciones problema planteadas en los trabajos prácticos de laboratorio.

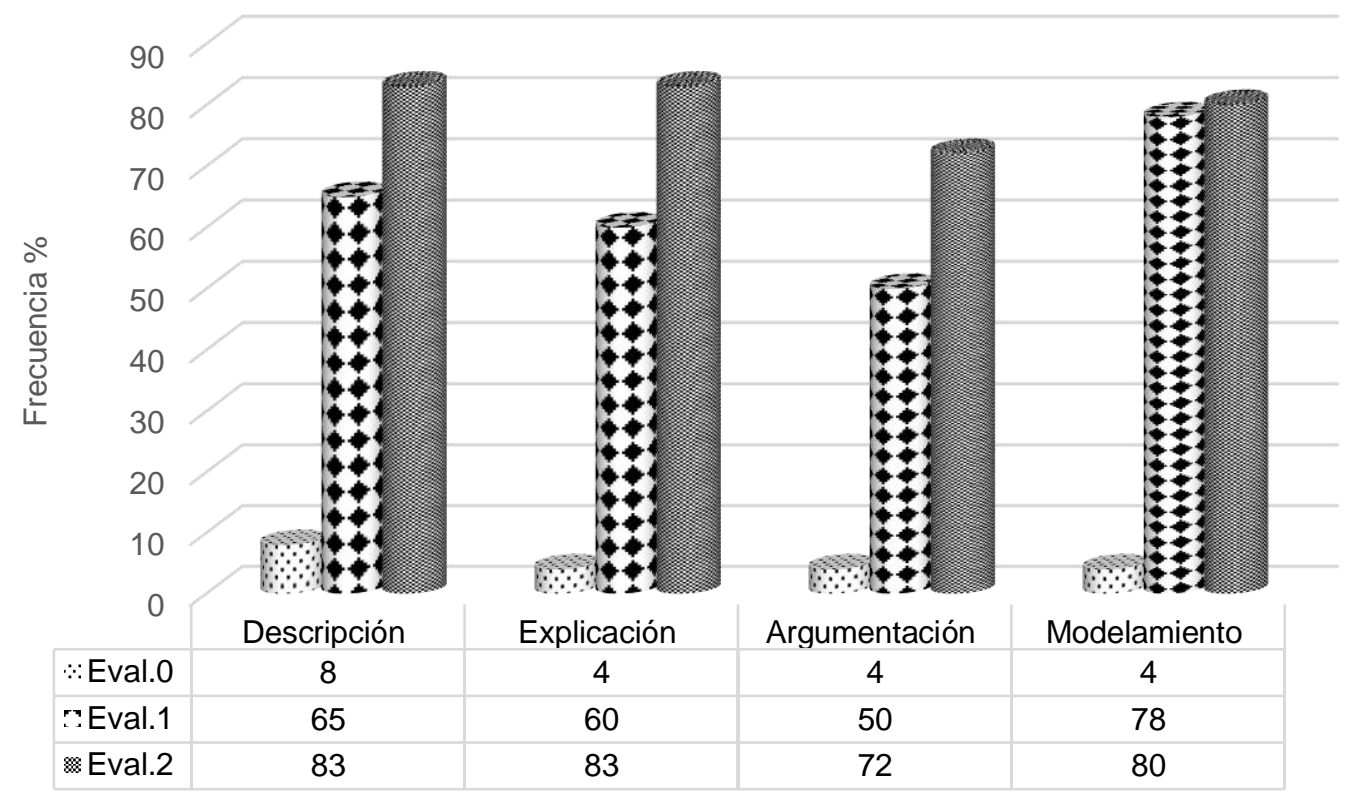

Fig. 4: Desarrollo de HCL en estudiantes de Ingeniería Civil en evaluaciones teóricas de física

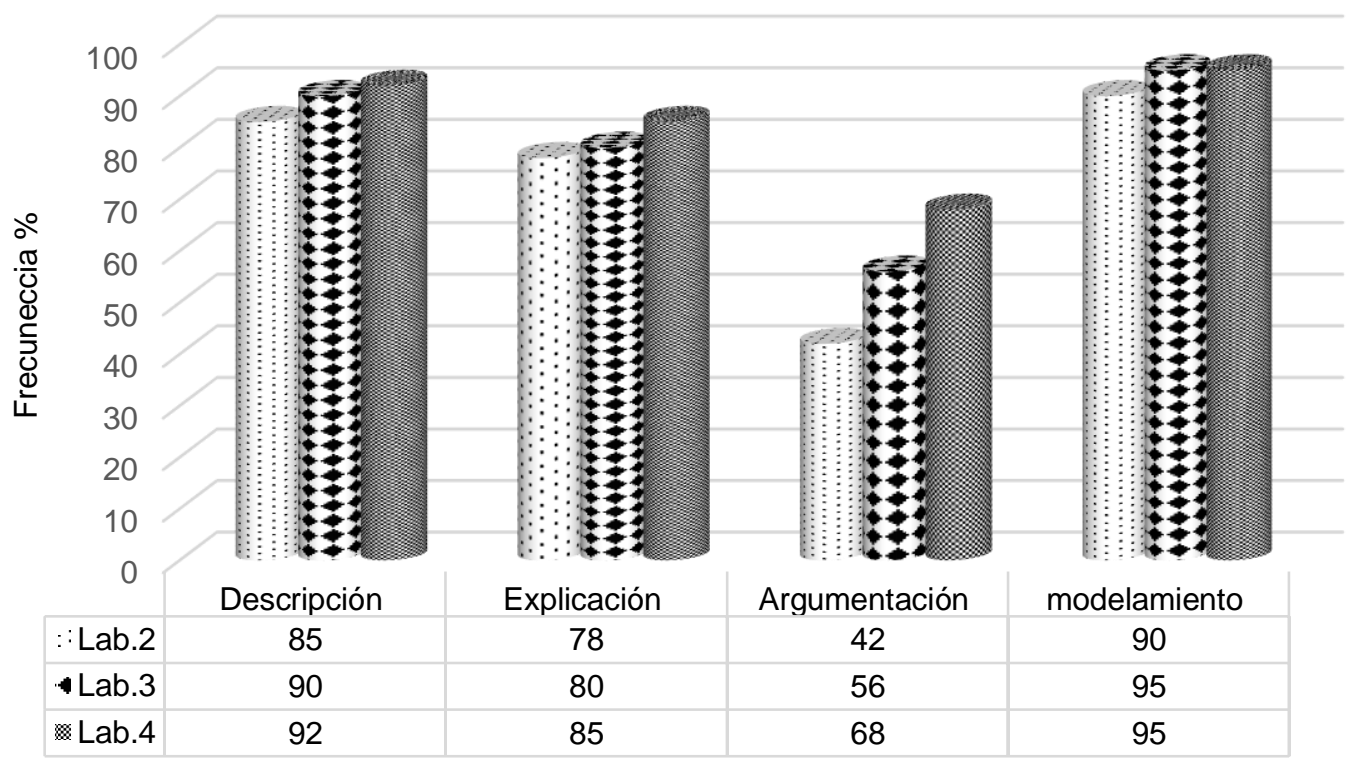

Fig.5: Desarrollo de HCL en estudiantes de Ingeniería en TPL de física 
En la Figura 6 se observa que en los TPL el nivel de desempeño en la habilidad de describir: en el módulo I muestra que un $43 \%$ de los alumnos alcanza el nivel de logro 2 adecuado referido a representar conocimientos físicos suficientes en la situación problema, y en el módulo II un $48 \%$ alcanza el nivel de logro avanzado al Identificar en el problema lo esencial de sus propiedades físicas.

En la habilidad de explicar en el módulo I el 52\% de los alumnos se encuentra en el nivel de logro 2 adecuado referido a los conocimientos de física que están representados en la situación problema y en el módulo II un $47 \%$ alcanza el nivel de logro avanzado emiten razones que evidencian la comprensión del problema. En la habilidad de argumentar en el módulo I y Modulo II un 52\% en $53 \%$ de los alumnos se encuentra en el nivel de logro 2 respectivamente, es decir, al enunciar razones con la intención de modificar su estado de conocimiento en relación con el corpus de conocimiento de física sobre la situación problema. Al modelar matemáticamente en el módulo I y módulo II un $49 \%$ y $47 \%$ de los alumnos se encuentra en el nivel de logro 2 adecuado respectivamente lo que implica hacer uso correcto del lenguaje matemático.

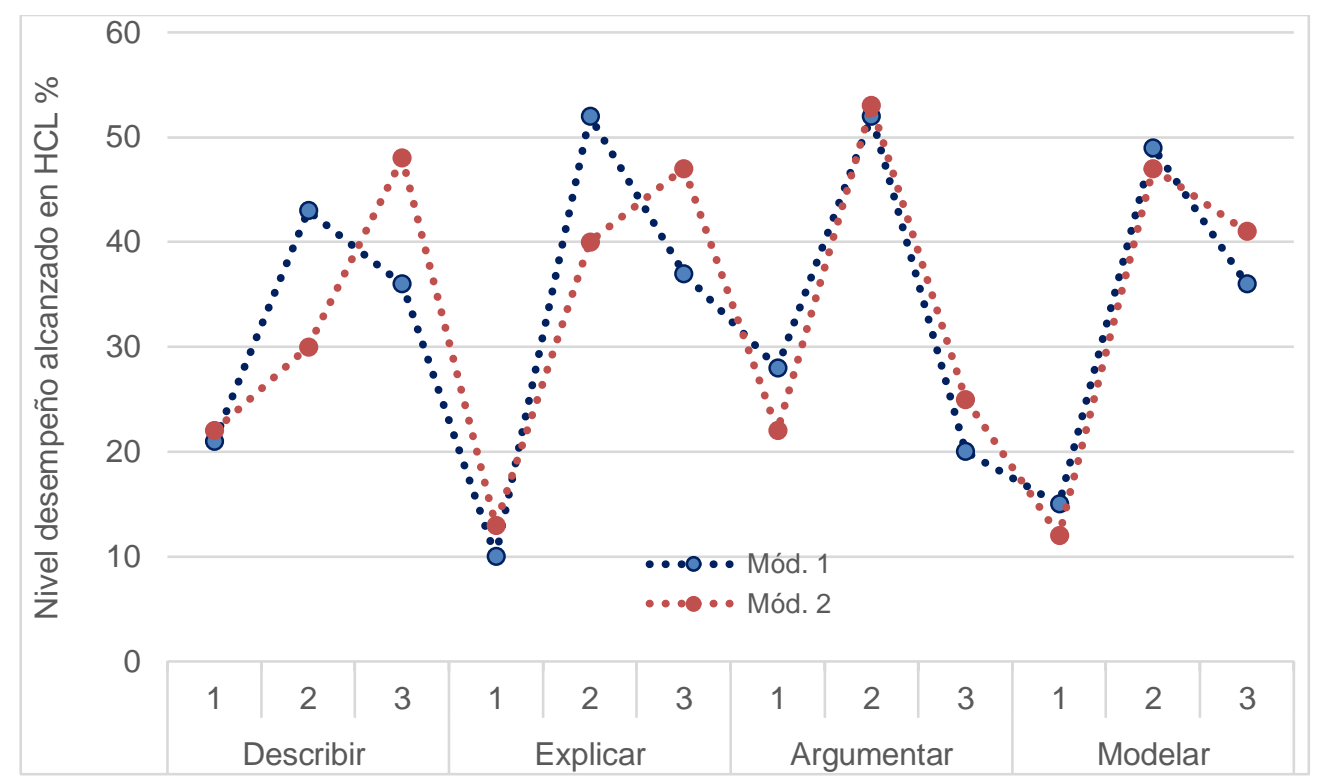

Fig. 6: Nivel desempeño alcanzado en HCL en estudiantes de Ingeniería en TPL de física

\section{DISCUSIÓN}

Los resultados obtenidos en esta investigación indican el impacto estadísticamente significativo ( $\mathrm{p}=0,000$; $99,99, \%$ ) de la resolución colaborativa de problemas en el rendimiento académico del GE en contraste a los dos GC. Tras haber realizado un análisis al rendimiento logrado, el incremento mayor se evidencia al finalizar el módulo II, lo que podría ser explicado por el significado que los estudiantes van otorgando al trabajo colaborativo para comprender el problema planteado, relacionar los conceptos asociados y consensuar sus explicaciones para tomar decisiones de su estrategia de solución, tanto en las clases teóricas como en los TPL. La propuesta didáctica implementada se aleja de la resolución mecánica (Pérez-Sánchez y PovedaSerra ,2008) y enseña a pensar con el propósito que los estudiantes desarrollen habilidades que involucran una cuidadosa planificación de las actividades y seguimiento sistemático de su aprendizaje por el profesor.

Por otra parte, la evaluación de las habilidades, es un proceso complejo que demanda la incorporación de una rúbrica para evaluar los niveles de logro que los estudiantes alcanzan al describir, explicar, argumentar y modelar matemáticamente cuando resuelven un problema, por tanto, con los datos obtenidos es posible afirmar que en teoría y TPL, los mayores niveles de logro corresponden a las habilidades concretos y simple (Jorba et al., 2000)de describir, explicar, las cuales usan los estudiantes para recordar una información ,aplicar conocimientos en situaciones triviales o para resolver ejercicios de menor demanda cognitiva (Zoller , 2013), por sobre la habilidad cognitivo lingüística complejo y abstracta, de argumentar, que implica a los estudiantes procesos cognitivos de orden superior relacionadas con la resolución de problemas y que utilizan para hacer conexiones y pensamiento de evaluación, que exigen una actividad intelectual más elaborada del estudiante.

Al contrastar el nivel de desempeño (desde el nivel de logro inadecuado, 2 adecuado y nivel 3 avanzado) alcanzado en el uso de las habilidades al resolver problemas entre el módulo I y II, sólo el uso de habilidad de explicar (47\%) alcanza el nivel avanzado en el módulo II y son variables en el nivel adecuado en las otras habilidades evaluadas. Estos resultados concuerdan con los encontrados por Córdoba et al., (2018) en el uso 
de las habilidades de explicaciones, seguidas de las opiniones, la justificación, la descripción y la argumentación en producciones comunicativas. Similar coincidencia, se evidenció en los estudiantes ante confusiones conceptuales en los TPL con una situación problema, donde se limitaban a opinar y casi no usar sus habilidades cognitivo-lingüísticas.

En este estudio se presentan evidencias de que la enseñanza de resolución de situaciones problemas de forma colaborativa permite que la mayoría de los estudiantes desarrolle las habilidades cognitivo lingüísticas de describir, explicar y modelar matemáticamente relacionadas con la interpretación del significado de los conceptos y leyes involucrados en los problemas de física y aumenta el rendimiento académico estadísticamente significativo en el GE respecto a los dos GC. La puesta en práctica de esta forma de trabajo en clases teóricas y prácticas no estuvo exenta de dificultades al inicio de la implementación, puesto que requiere de los estudiantes trabajo colaborativo con una participación activa en la comprensión mutua del problema para describirlo, discutir la información y los datos para conectar sus ideas previas para llegar a una explicación, lo que involucra y actitudes de respeto por las ideas del otro, al dialogar y discutir sus estrategias de solución . Los alcances logrados con la aplicación del cambio metodológico están de acuerdo con la literatura al señalar que a medida que mejora el trabajo en equipo, mejoran las habilidades colaborativas y sobre todo los estudiantes son capaces de transmitir lo aprendido de forma oral y por escrito.

Sin embargo, el estudio permitió develar las dificultades de los estudiantes en la habilidad de argumentación que corresponden a procesos cognitivos de orden superior, por sobre las habilidades concretas como describir, explicar. En la revisión de los argumentos se estableció que carecen de razones que relacionen: evidencias, explicaciones en coherencia con los juicios de valor expresados. En este sentido, sería conveniente enseñar explícitamente a emplear las habilidades cognitivo lingüísticas de forma progresiva, aplicándolas en aspectos conceptuales cada vez más complejos y abstractos.

Esta forma de enfocarse al resolver los problemas podría reemplazar la estrategia tradicional de los estudiantes de buscar los datos para recurrir a una fórmula y a la instrucción de conocimiento unidireccional del docente/libro de texto, la que ha resultado insuficiente en el logro de aprendizaje de los dos grupos control. Aprender colaborativamente a resolver, situaciones problemas es aprender física, dando la oportunidad para que el estudiante se exprese, describiendo, explicando, argumentando a partir de sus ideas previas, la interacción entre sus pares y con el profesor permite cuestionar y observar cómo el conocimiento emerge en el contexto del problema teórico o desde su experimentación, además de poder resignificarlos, por lo que es susceptible de ser aplicado en otras asignaturas.

\section{CONCLUSIONES}

De acuerdo al trabajo presentado y a los resultados obtenidos, se pueden plantear las siguientes aseveraciones de conocimiento:

La presente investigación es de gran relevancia al enseñar y aprender física, ya que considera las habilidades cognitivo lingüísticas en la resolución de problemas, incorporando proceso metacognitivos que usa el estudiante para comprender, resolver los ejercicios y problemas, para asociarlos a su realidad, dejando de lado fórmulas matemáticas, que es, una dificultad para aprender física en los primeros años de Universidad. En este contexto, se observó que la secuencia de actividades de aprendizaje implementada en el grupo experimental obtuvo un mayor rendimiento académico en la teoría que los dos grupos controles, y en menor medida en los trabajos prácticos de laboratorio donde se trabaja en equipo y en la disposición para adaptarse a trabajar con las habilidades cognitivo lingüísticas describir, explicar y el modelado matemático.

Los estudiantes desarrollaron el modelado matemático por medio del uso de la integral y derivada como método único para resolver los problemas de cinemática, que permite obtener las ecuaciones de movimiento y elimina el uso indiscriminado y reiterativo de fórmulas, lo que promueve la interpretación física del problema en cuestión, y requiere de un desempeño abstracto complejo,

Los resultados evidencian que los estudiantes en general presentaron dificultades para argumentar, lo que sugiere una adaptación de la propuesta didáctica en relación al tiempo y mediación del docente en las actividades de aprendizaje de argumentación. Aquí se abre una brecha para nuevas investigaciones, que puede incorporar otras variables para la mejora de los resultados: la predicción y su relación con el modelamiento, dialogo de los protagonistas, lo que evidencia lo incipiente de esta línea de investigación.

\section{AGRADECIMIENTOS}

El presente trabajo es parte de una investigación, que es posible gracias al financiamiento logrado a través del Proyecto de Investigación FONDECYT №1181525. 


\section{REFERENCIAS}

Adams, W.K, y Wieman, C. E, Analyzing the many skills involved in solving complex physics problems. American Journal of Physics, 83(5), 459-467. (2015).

Aragón, M.M., Las Ciencias Experimentales y la Enseñanza Bilingüe. Revista Eureka sobre Enseñanza y Divulgación de las ciencias, 4(1),152-175. (2007).

Cohen, L., Manion L., Método de investigación educativa. La Muralla, Madrid, España (1990).

Córdoba, F., Castelblanco, J.L y. García-Martínez Á., Desarrollo de las habilidades cognitivo-lingüísticas en ciencias bajo la modalidad de educación virtual a distancia. doi: 10.5565/rev/ensciencias.2189, Enseñanza de las ciencias, 36(3), 163178. (2018).

Couto, P., García-Barros, S. y Martínez- Losada, C., Cómo son las actividades de didáctica de las ciencias que proponemos a los futuros maestros de primaria. Enseñanza de las ciencias: revista de investigación y experiencias didácticas, (Extra), 877-882. (2013).

Docktor, J.L; Strand, N.E; Mestre J.P. y Ross, B. H., Conceptual problem solving in high school physics. doi: 10.1103/PhysRevSTPER.11.020106, Physical Review Special Topics-Physics Education Research, $11(2), 020106$. (2015).

Docktor, J.L., Dornfeld, J., y otros siete autores., Assessing student written problem solutions: A problem-solving rubric with application to introductory physics. Physical review physics education research, 12(1), 010130. (2016).

Duit, R., Schecker, H., y otros dos autores., Handbook of research on science education, V.II, Teaching Physics, 434-456, Routledge, New York, United States (2014).

García- Barros, S. y Martínez Losada, C., La importancia de las habilidades cognitivo-lingüísticas asociadas al estudio de la Astronomía desde la perspectiva del profesorado. Enseñanza de las Ciencias, 32(1), 179-197. (2014).

García Peñalvo, F. y Colomo Palacios, R., Innovative teaching methods in Engineering. Engineering Education (IJEE), 31(3), 689-693. (2015).

Guisasola, J., Ceberio, M. y Zubimendi, J., University students' strategies for constructing hypothesis when tackling paperand-pencil tasks in physics. Research in Science Education, 36(3), 163-186. (2006).

Johnson, D.W., Johnson, R.T., y otros dos autores, The new circles of learning: Cooperation in the classroom and school. ASCD. Washington, United States (1994).

Jorba, J., Gómez, I. y Prat, À., Hablar y escribir para aprender: uso de la lengua en situación de enseñanza-aprendizaje desde las áreas curriculares. 29-49. Barcelona: ICE Universitat Autònoma de Barcelona. Síntesis, España (2000).

Khasanah, N., Wartono, W. y Yuliati, L., Analysis of Mental Model of Students Using Isomorphic Problems in Dynamics of Rotational Motion Topic. Jurnal Pendidikan IPA Indonesia, 5(2), 186-191. (2016).

Kuhn, L. y Reiser, B., Structuring activities to foster argumentative discourse. In annual meeting of the American Educational Research Association, San Francisco, United States. (2006).

Lamon, S., Parker, W. A. y Houston, S.k., Modelado matemático: una forma de vida-ICTMA 11. Elsevier, Wisconsin, United States (2003).

Lawson, A. E., Sound and faulty arguments generated by preservice biology teachers when testing hypotheses involving unobservable entities. Journal of Research in Science Teaching: The Official Journal of the National Association for Research in Science Teaching, 39(3), 237-252. (2002).

Mayer Foulkes D., López, M.F. y Servan, E., Habilidades Cognitivas: Transmision Intergeneracional Por Niveles Socioeconomicos. (N. 7180). University Library of Munich, Germany. (2008).

Nersessian, N.J., Should physicists Preach what they Practice? Science \& Education, 4(3), 203-226. (1995)

OECD., PISA 2015 Assessment and Analytical Framework: Science, Reading, Mathematic, Financial Literacy and Collaborative Problem Solving. Paris: OCDE. (2015).

Osborne, J., Erduran, S. y Simon, S., Mejora de la calidad de la argumentación en la ciencia escolar. Revista de investigación en la enseñanza de las ciencias, 41(10), 994-1020. (2004).

Perales, J., y. Cañal de León, P., Didáctica de las Ciencias: Teoría y Práctica de la Enseñanza de las Ciencias. Marfil: Alcoy. España (2000).

Perales, F.J., La resolución de problemas en física. Revista Eureka sobre Enseñanza y Divulgación de las Ciencias, 3(3) 524-525. (2006).

Pérez-Sánchez, A. M. y Poveda-Serra, P., Efectos del aprendizaje cooperativo en la adaptación escolar. Revista de Investigación educativa, 26(1), 73-94. (2008).

Pirela, J. y Cortés, J.D.J., El desarrollo de competencias informacionales en estudiantes universitarios: Experiencia y perspectivas en dos universidades latinoamericanas. Investigación bibliotecológica, 28(64), 145-172. (2014).

Pulgar, J., Candia, C., y Leonardi, P., Social networks and academic performance in physics: Undergraduate cooperation enhances ill-structured problem elaboration and inhibits well-structured problem solving. Phys. Rev. Phys. Ed. Res. (2020). 
Ramos, A.I., Herrera, J.A. y Ramírez, M.S., Desarrollo de habilidades cognitivas con aprendizaje móvil: un estudio de casos. Revista Comunicar, (34), 201-209. (2010).

Reed, S.K., Cognition. Theory and Applications. Thomson Wadsworth, United States (2007).

Sánchez, I.R, Pulgar, J.A. y Flores, P., The Impact of Using Derivatives and Integrals in Learning Kinematics at the University. International Journal of Review in Applied and Social Sciences. 1(7), 23-34. (2015).

Sandoval, A. y Franchi, L., Meta comprensión en estudiantes de ingeniería. Omnia, ISSN:1315-8856, 13(2), 98-119. (2007).

Sandoval, W. y Millwood, K., The quality of students' use of evidence in written scientific explanations. Cognition and instruction, 23(1), 23-55. (2005).

Schmal, R., Evolución de un Programa de Formación en Competencias Genéricas, Formación Universitaria, 8(6), 95-106 (2015).

Soares, F. O., Sepúlveda, M.J; Monteiro, S. Lima, R.M. y otro autor, An integrated project of entrepreneurship and innovation in engineering education. Mechatronics, 23(8), 987-996 (2013).

Solbes, J., Montserrat, R. y Furió, C., El desinterés del alumnado hacia el aprendizaje de la ciencia: implicaciones en su enseñanza. Didáctica de las Ciencias Experimentales y Sociales. (21), 91-117. (2007).

Supriyanti, F.M.T., Hernani, H. y Mulyanti, S., The use of problem-solving model in the material of the genetic information flow to improve the students'concept mastery. Jurnal Pendidikan IPA Indonesia, 4(1), 73-78. (2015).

Truyol, M.E., Sanjosé López, V. y Gangoso, Z.E., Obstacles modelling reality: Two exploratory studies on physics defined and undefined problems. Journal of Baltic Science Education, 2014, 13(6), 883-895. (2014).

Zohar, A., y Nemet, F., Fostering students' knowledge and argumentation skills through dilemmas in human genetics. Journal of Research in Science Teaching: The Official Journal of the National Association for Research in Science Teaching, 39(1), 35-62. (2002).

Zoller, U., Alfabetización en ciencia, tecnología, medio ambiente, sociedad (STES) para la sostenibilidad: ¿qué debería tomar en la educación química / científica? Educación Química, 24 (2), 207-214. (2013). 